LA-UR- $11-03407$

Approved for public release;

distribution is unlimited.

Title:

A stable hyperelastic model for foamed rubber

Author(s):

Matthew W. Lewis and Partha Rangaswamy, W-13

Intended for:

7th European Conference on Constitutive Models for Rubber Dublin, Ireland

20-23 September, 2011

- Los Alamos

NATIONAL LABORATORY

EST. 1943

\begin{abstract}
Los Alamos National Laboratory, an affirmative action/equal opportunity employer, is operated by the Los Alamos National Security, LLC for the National Nuclear Security Administration of the U.S. Department of Energy under contract DE-AC52-06NA25396. By acceptance of this article, the publisher recognizes that the U.S. Government retains a nonexclusive, royalty-free license to publish or reproduce the published form of this contribution, or to allow others to do so, for U.S. Government purposes. Los Alamos National Laboratory requests that the publisher identify this article as work performed under the auspices of the U.S. Department of Energy. Los Alamos National Laboratory strongly supports academic freedom and a researcher's right to publish; as an institution, however, the Laboratory does not endorse the viewpoint of a publication or guarantee its technical correctness.
\end{abstract}

Form $836(7 / 06)$ 


\title{
A stable hyperelastic model for foamed rubber
}

\author{
M.W. Lewis \& P. Rangaswamy \\ Los Alamos National Laboratory, Los Alamos, New Mexico, USA
}

\begin{abstract}
A hyperelastic strain energy function for foamed rubber that is based on the physical features of a typical uniaxial compression curve for foams and the behavior of a spherical pore in a spherical, incompressible Mooney-Rivlin matrix is presented. The model is unconditionally stable when positive moduli are used, and can represent most foam test data, including variable Poisson behavior during compression. This most general model has six parameters, including four moduli, a dimensionless parameter associated with buckling or plateau strain, and an initial porosity. The model will be described and its ability to fit compression response data for PDMS foams over a range of relative densities will be evaluated. Several potential extensions of this model will be discussed, including representation of the Mullins effect, representation of aging behavior, inclusion of matrix compressibility, and modeling the effect of pore gas response.
\end{abstract}

\section{INRODUCTION}

Elastomeric foams, or foamed rubbers, comprise a class of materials that are useful in many engineering applications. Because of their relatively low densities, they offer benefits at low component weight. Because of low initial stiffness and ability to undergo large, fully recoverable elastic deformations, the materials work well as impulse limiters and cushions that lower stresses that result from differential thermal expansion or contraction of nested components.

Current efforts at mechanical modeling of foamed rubbers can be divided into two approaches. The first of these approaches consists of attempts to understand the mechanics of foam deformation by modeling realizations of unit cells or larger volumes of foam with mesh discretizations that represent actual porosity and matrix morphology. The second of these approaches consists of phenomenologicallybased constitutive model development, fitting, and evaluation.

The first of these approaches, which may be seen in work by Kraynik et al. (1998), Braydon et al. (2005), and a few others, will be referred here to as Direct Numerical Simulation (DNS) investigations. These DNS investigations are often limited by mesh resolution, mesh distortion, and contact mechanics issues. Additionally, the process of moving from DNS to component- or system-level modeling is not yet developed well.
The second of these approaches, of which the current work is an example, is represented by the work of Jemiolo and Turtletaub (2000). The most commonly used hyperelastic model for foamed rubbers in finite element analysis is the model proposed by Jemiolo and Turtletaub, a generalization of the Ogden (1984) strain energy function in isochoric principal stretches. That model has the advantage that it can reproduce test data with good accuracy. It has disadvantages that include possible instability and high sensitivity of model fit parameters to data perturbation and fitting procedure. As a result, it is impractical to represent variability in mechanical behavior with model parameter distributions. The work of Danielsson et al. (2004) is also an example of a developed hyperelastic model for these materials, but is limited to lower porosity foams as it does not capture the classic plateau behavior observed in foams with low relative density.

This work describes a proposed new model for foamed rubber. The model was developed to provide both accurate fits to test data and model parameters that can be varied sensibly to represent material variability. The model consists of a strain energy function that was manufactured to reproduce foam compression behavior at moderate porosities, namely an initial Poisson's ratio, a nearly linear region at small strains, an inflection point where the material tangent compressive modulus drops significantly, and a strongly stiffening region as porosity is eliminated. These first three features were intro- 
duced phenomenologically, that is without a micromechanical motivation.

The stiffening region response was derived using an approach similar to Danielsson et al. (2004) for evaluating the strain energy of a void in an incompressible material subjected to far-field hydrostatic compression and a volume-conserving deformation. Our only extension to this part of the model is the calculation of the integrated second isochoric invariant for this deformation.

This model has been coded as a material model subroutine for use with a non-linear static finite element program and has been used to model a validation problem involving large compressive strains combined with substantial torsional deformation.

The following sections of this paper consist of a model description section detailing the form of the model and the parameter fitting process used here, an example section in which the model is shown to reasonably fit uniaxial strain compression test data, a model extensions section in which extensions to the model are considered and simple aging and pore gas compression extensions are demonstrated, and a conclusions section.

\section{MODEL DESCRIPTION}

Before we begin the discussion of the phenomenology of foamed rubber, a few words about nomenclature and conventions are appropriate. When foams are compressed, large compressive strains can obtain and significant volume change occurs. We therefore need to be very specific about our choices of stress and strain measures. Note that if an experimentalist refers to "true stress" when working with foams in compression, he or she had better have measured lateral strain, because standard assumptions of incompressibility at large deformations associated with metals and solid elastomers do not apply to foams. For our purposes, we will refer to engineering stress and engineering strain, both used here as positive in compression. It should also be noted that the present work is primarily focused on foam response under combined loads of compression and shear. The theory developed extends to tension, but most foams are not useful in tension as they are very weak because of their porosity.

\subsection{Phenomenology}

In much of the literature on foams, foam response is characterized in terms of the relative density of the foam, $\rho^{*}$. The relative density of a foam is defined as the ratio of the density of the foam, $\rho_{f}$, to the density of the parent solid, $\rho_{s}$. The initial porosity of the foam, $\phi_{o}$, is related to the relative density as follows:

$\phi_{o}=1-\rho^{*}=\frac{\rho_{s}-\rho_{f}}{\rho_{s}}$.

A plot of uniaxial stress in compression vs. axial strain for monotonic loading of foam with a relative density of approximately 0.37 is shown in Figure 1. The linear, plateau-like, and densification regions are clearly indicated. A hyperelastic material subjected to this loading would unload along the same curve to a state of no strain when unstressed. A real foamed elastomer would exhibit some hysteresis and possibly cyclic softening. For our purposes, we ignore these dissipative mechanisms, with the understanding that they can be added to a stable, energyconserving model.

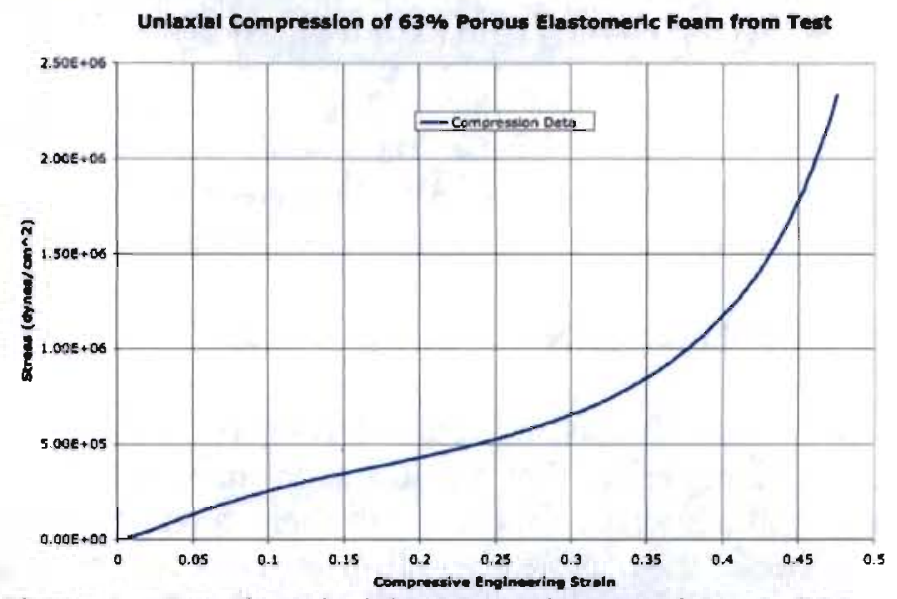

Figure 1. Sample uniaxial compression test data on PDMS foam with an initial porosity of approximately 0.63 .

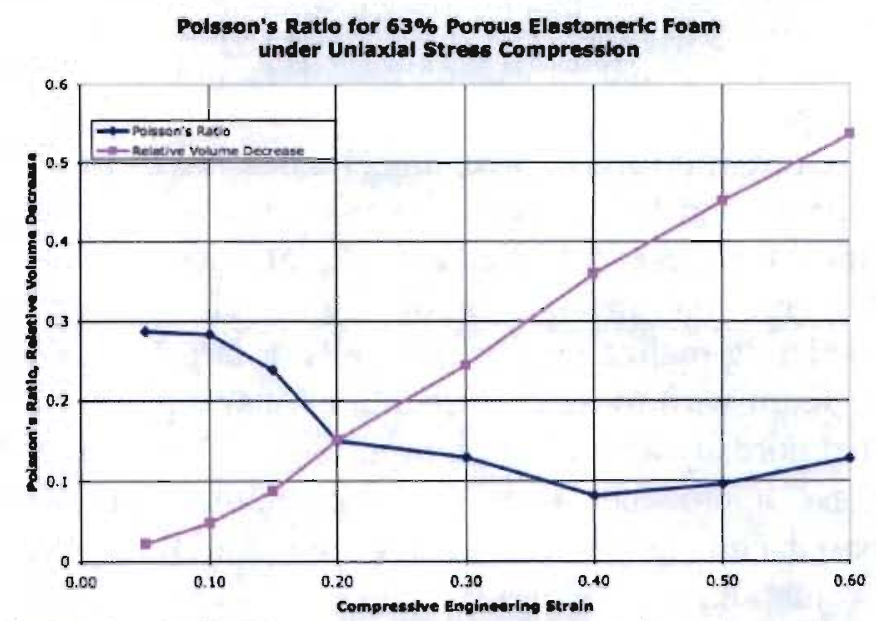

Figure 2. A plot showing the nonincremental version of Poisson's ratio and the relative volume decrease measured in a lubricated uniaxial stress compression test of a PDMS foam with an approximate initial porosity of 0.63 .

Figure 2 is a plot of the nonincremental form of Poisson's ratio, here defined as the ratio of the lateral engineering strain to the compressive axial engineering strain in a uniaxial stress compression test, as measured by Mooday (2002). These data suggest 
that the volumetric stiffness drops significantly in the plateau-like region of the compression stressstrain curve. The relative volume change, $1-J$, where $J$ is the relative volume or the determinant of the deformation gradient, is also plotted in Fig. 2. During densification, the volumetric response stiffens, until the nearly incompressible behavior of the parent elastomer is approached.

\subsection{The Model}

We have chosen to break the total strain energy function into two main terms to capture the salient features discussed previously.

\subsubsection{Initial stiffness and abrupt stiffness decrease}

In order to describe the initial abrupt decrease in volumetric stiffness discussed previously, we have chosen to use a simple hyperelastic model based on linear uncoupled stress response in principal stretches added to a volumetric strain response that is quadratic in $(J-1)$ until the relative volume hits a critical point, $J_{b}$, at which point the function becomes linear. This part of the strain energy function is as follows:

$$
\begin{aligned}
& U_{i p}=\frac{\hat{E}}{2} \sum_{i=1}^{3}\left(\lambda_{i}-1\right)^{2} \\
& +\hat{K}\left\{\left(J_{b}-1\right)\left(J-\frac{J_{b}+1}{2}\right)+\mathrm{H}\left[J-J_{b}\right]\left[\frac{(J-1)^{2}}{2}-\left(J_{b}-1\right)\left(J-\frac{J_{b}+1}{2}\right)\right]\right\}
\end{aligned}
$$

In Equation 2 above, $\hat{E}$ is an uncoupled version of Young's modulus (i.e. if the uncoupled bulk modulus were zero), $\lambda_{I}$ are the principal stretches, and $\hat{K}$ is the uncoupled bulk modulus (i.e. if the uncoupled Young's modulus were zero). The expression $\mathrm{H}[x]$ is the unit step function at $x$. The other terms in Equation 2 have been defined previously in this article.

\subsubsection{Micromechanically-Based Hyperelastic Model for Compaction}

In order to describe the substantial increase in stiffness and stress as the compressive strain approaches the initial porosity of a foam, we borrow from a strain energy approach used by Danielsson et al. (2004) for porous rubber. We have generalized their approach to include both first and second isochoric invariants in the strain energy function for an isolated void enclosed in a spherical shell of incompressible, Mooney-Rivlin material. The strain energy expression is as follows:

$U_{p c}=C_{10}\left(\hat{\bar{I}}_{1}-3\right)+C_{01}\left(\hat{\bar{I}}_{2}-3\right)$.

In Equation 3, $C_{10}$ and $C_{01}$ are the Mooney-Rivlin coefficients of the parent material, and the other two unfamiliar symbols represent the generalized isochoric invariants as follows:
$\vec{I}_{1}=I_{1} f_{1}(J)+3 \phi_{o}$

where

$f_{1}(J)=\frac{2 J-1}{J^{1 / 3}}+\left(2-2 J-\phi_{o}\right)\left[\frac{\phi_{o}}{J-\left(1-\phi_{o}\right)}\right]^{1 / 3}$,

and

$\hat{\bar{I}}_{2}=\bar{I}_{2} f_{2}(J)+3 \phi_{o}$.

where

$f_{2}(J)=J^{1 / 3}(2-J)+\frac{\left(J-1-\phi_{o}\right)\left[J-\left(1-\phi_{o}\right)\right]^{1 / 3}}{\phi_{o}^{1 / 3}}$

In Equations $4 \mathrm{a}$ and $5 \mathrm{a}$, the first and second isochoric invariants have been used. These invariants are defined as follows:

$\bar{I}_{1}=\bar{B}_{i i}$

and

$\bar{I}_{2}=\bar{B}_{i i}^{-1}$,

where

$\overline{\mathbf{B}}=J^{-2 / 3} \mathbf{B}$,

and

$\mathbf{B}=\mathbf{F} \cdot \mathbf{F}^{T}$,

or

$\bar{B}_{i j}=J^{-2 / 3} F_{i k} F_{k j}$

In Equations 9 and 10, $\mathbf{F}$ is the deformation gradient, or the derivative of the current configuration with respect to the reference configuration.

\subsubsection{Model Summary}

The full constitutive model proposed, then, can be summarized as a hyperelastic model with a strain energy function that is expressed as follows:

$U=U_{l p}+U_{p c}$.

The Cauchy stress, $\boldsymbol{\sigma}$, can be derived as follows:

$\boldsymbol{\sigma}=\frac{1}{J} \frac{\partial U}{\partial \mathbf{F}} \cdot \mathbf{F}^{T}$.

More explicitly, the Cauchy stress is as follows:

$$
\begin{aligned}
\boldsymbol{\sigma} & =\frac{\hat{E}}{J} \sum_{i=1}^{3} \lambda_{i}\left(\lambda_{i}-1\right) \mathbf{p}_{i} \otimes \mathbf{p}_{i}+\hat{K}\left\{J_{b}-1+\left\langle J-J_{b}\right\rangle\right\} \mathbf{i} \\
& +C_{10}\left[\frac{2}{J} f_{1}(J) \operatorname{dev}(\overline{\mathbf{B}})+\bar{I}_{1} f_{1}^{\prime}(J) \mathbf{i}\right] \\
& +C_{01}\left[\frac{2}{J} f_{2}(J) \operatorname{dev}\left(\bar{I}_{1} \overline{\mathbf{B}}-\overline{\mathbf{B}}^{2}\right)+\bar{I}_{2} f_{2}^{\prime}(J) \mathbf{i}\right]
\end{aligned}
$$


In Equation 13, the vectors $\mathbf{p}_{i}$ are unit principal directions vectors associated with the corresponding principal stretches $\lambda_{i}, \operatorname{dev}(\mathbf{A})$ signifies the deviatoric (traceless) part of the second order tensor $\mathbf{A}$, and $\mathbf{i}$ is the second order identity tensor. The prime symbol, ()$^{\prime}$, denotes differentiation of a function with respect to its argument. The other symbols in Equation 13 should have been adequately explained at this point, except for the Macaulay brackets, $\langle\ldots\rangle$, which provide the value of the term inside the brackets if it is positive, and zero otherwise.

\section{EXAMPLE}

In this section, we consider uniaxial strain compression. This consideration is motivated by the fact that for many cushioning applications, foams are made very thin. As a result, compression tests on these materials tend to be in the thin direction and end effects are not negligible. The specimens tend to be in a condition of near uniaxial strain.

In uniaxial strain, only one stretch is not unity. Let the axial stretch be $\lambda$. The relative volume, $J$, is then also $\lambda$. The only direction in which the stress can be easily measured is the axial direction, and it can be shown that the axial stress can be represented by a combination of linearly independent functions of axial stretch with the model moduli as coefficients.

Provided that one has values for the initial porosity, $\phi_{o}$, and for the buckling point, $J_{b}$, one may fit the moduli using a linear least squares approach.

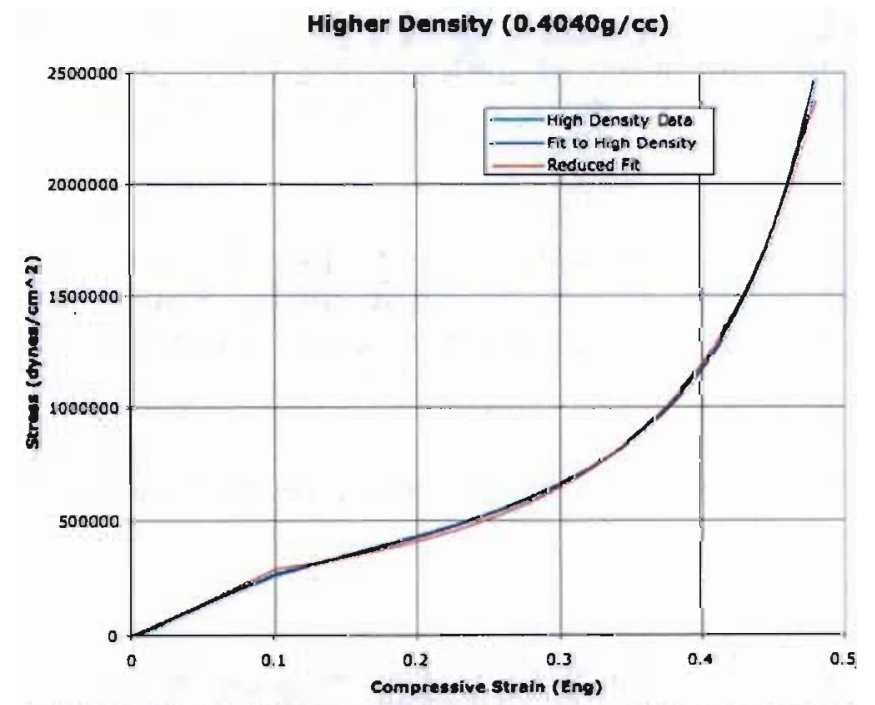

Figure 3. Uniaxial strain compression data and fits to PDMS foam with a density of $0.4040 \mathrm{~g} / \mathrm{cc}$, an approximate initial porosity of 0.63 . The reduced fit is one for which $C_{01}$ has been set to zero.

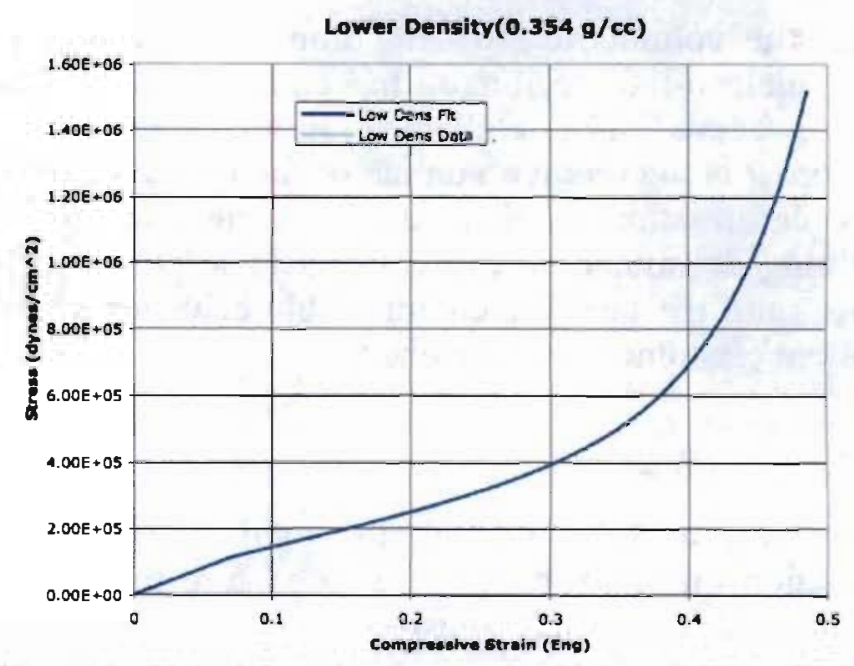

Figure 4. Uniaxial strain compression data and fits to PDMS foam with a density of $0.354 \mathrm{~g} / \mathrm{cc}$, an approximate initial porosity of 0.68 .

In Figures 3 and 4, model fits to uniaxial strain compression data for foams with porosities of 0.63 and 0.68 , respectively, are presented. It should be noted that the tests used to generate these two figures were not true uniaxial strain tests, but were rather unconfined compression tests with very low $\mathrm{L} / \mathrm{D}$ ratios, which were approximately 0.025 .

\section{MODEL EXTENSIONS}

In this section we consider two main model extensions. The first is associated with material aging in the form of changing crosslinks under a defined deformation. The second is associated with the effects of pore gas compression.

\subsection{Material aging}

We consider here the effect aging under an imposed deformation. The approach here is based on work of Tobolsky (1960). We consider that the material in the reference state is initially stress free and its network of polymer chains and that the mechanical response of its physical and chemical crosslinks is well described by a particular strain energy function (here chosen as our model for foams).

The material is then deformed to some new configuration. In this new configuration, the storage configuration, new crosslinks are formed during an aging process. Old crosslinks may break, too. The new set of crosslinks are envisioned as forming a second network that is stressless in the configuration in which they formed. The mechanical response of this second network is assumed to be well represented by a strain energy function similar to that of the original material, but the reference state for the second network is the one under which it was formed. 
Let us call the deformation gradient that describes the deformation of the material under which this type of aging occurs $\mathbf{F}_{s}$. The relative deformation gradient, $\mathbf{F}_{r}$, for the second network is found using a multiplicative decomposition of the total deformation gradient as follows:

$$
\mathbf{F}=\mathbf{F}_{r} \cdot \mathbf{F}_{s} \text {, }
$$

or

$$
\mathbf{F}_{r}=\mathbf{F} \cdot \mathbf{F}_{s}^{-1} \text {. }
$$

Similarly, one can construct all the relevant tensors and invariants pertinent to the strain energy function based on this relative deformation tensor. The relative volume as measured from the storage state is as follows:

$$
J_{r}=\frac{J}{J_{s}} .
$$

Based on incompressibility of the parent material, we can derive a new initial porosity for the storage state as follows:

$$
\phi_{o}^{r}=1-\frac{1-\phi_{o}}{J_{s}} \text {. }
$$

\subsubsection{Application of aging model to uniaxial strain}

Let us consider using the approach outlined above to describe a material subjected to a uniaxial strain compression storage condition at a strain well beyond the buckling point (stress-strain slope decrease point). Furthermore we will consider that the virgin material is well described with $C_{01}=0$.

The mechanical response of the second network about the storage configuration will be assumed for demonstration purposes to be described well by the first isochoric invariant part of the strain energy function as follows:

$U_{2}=C_{10}^{r}\left(\hat{\bar{I}}_{1}^{r}-3\right)$,

where

$\hat{\bar{I}}_{1}^{r}=\bar{I}_{1}^{r} f_{1}^{r}\left(J_{r}\right)+3 \phi_{o}^{r}$

where

$$
f_{1}^{r}\left(J_{r}\right)=\frac{2 J_{r}-1}{J_{r}^{1 / 3}}+\left(2-2 J_{r}-\phi_{o}^{r}\right)\left[\frac{\phi_{o}^{r}}{J_{r}-\left(1-\phi_{o}^{r}\right)}\right]^{1 / 3} .
$$

We could consider that the moduli for the strain energy network of the initial network change also, but for demonstration purposes we will consider them constant. The resulting strain energy density function is as follows:

$U=U_{l p}+U_{p c}+J_{s} C_{10}^{r}\left(\hat{\bar{I}}_{1}^{r}-3\right)$
We now consider the uniaxial strain mechanical response of the new material as follows. The Cauchy stress for this aged material is then as follows:

$$
\begin{aligned}
\boldsymbol{\sigma} & =\frac{\hat{E}}{J} \sum_{i=1}^{3} \lambda_{i}\left(\lambda_{i}-1\right) \mathbf{p}_{i} \otimes \mathbf{p}_{i}+\hat{K}\left\{J_{b}-1+\left\langle J-J_{b}\right\rangle\right\} \mathbf{i} \\
& +C_{10}\left[\frac{2}{J} f_{1}(J) \operatorname{dev}(\overline{\mathbf{B}})+\bar{I}_{1} f_{1}^{\prime}(J) \mathbf{i}\right] \\
& +C_{10}^{r} J_{s}\left[\frac{2}{J_{r}} f_{1}^{r}\left(J_{r}\right) \operatorname{dev}\left(\overline{\mathbf{B}}_{r}\right)+\bar{I}^{r}\left(f_{1}^{r}\right)^{\prime}\left(J_{r}\right) \mathbf{i}\right]
\end{aligned}
$$

The axial stress under conditions of uniaxial strain is again a combination of linearly independent functions multiplied by the moduli of the model.

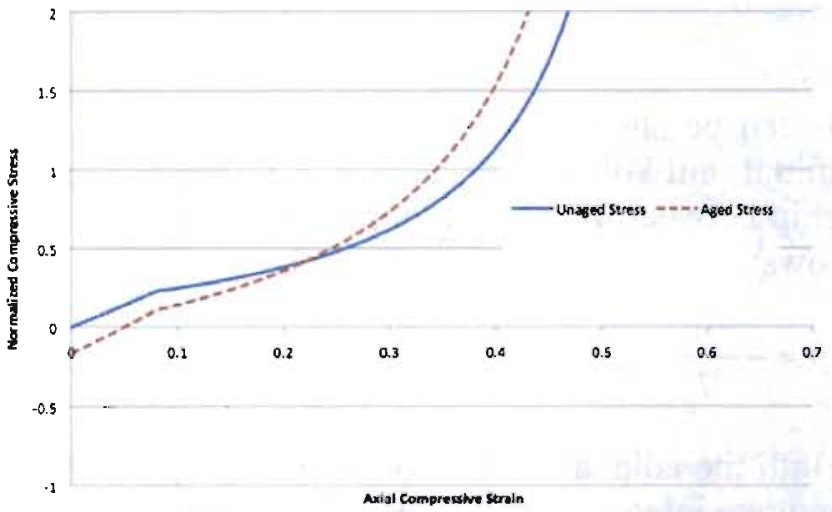

Figure 5. Example normalized compressive stress curves for an unaged material and one stored at $22 \%$ axial strain compression.

A sample plot showing the unaged and aged uniaxial deformation response for a fictitious $62 \%$ porous rubber material aged under an imposed compressive strain of $22 \%$ is shown in Figure 5 . In this case, the $C_{10}$ moduli for the second network and the initial network are identical.

\subsection{Pore gas effects}

Let us consider the two different relationships for the compression of gases. The first is an isothermal treatment where it is assumed that the ambient environment can easily exchange heat with the gas while it is compressed so that the gas is always at the ambient temperature. Another way of saying this is that the compression is slow compared to heat transfer processes and that the heat generated in compression is not enough to raise the temperature of the environment.

The pressure under these isothermal conditions is as follows:

$p_{i}=p_{o} \frac{v_{o}}{v}$

where $p_{i}$ is the current pressure, $v$ is the current volume, $p_{0}$ is the initial pressure, and $v_{o}$ is the initial 
volume. We can express this in terms of the relative volume of the gas, $J_{g}$, as follows:

$$
p_{i}=\frac{p_{o}}{J_{g}} .
$$

$J_{g}$ is defined as follows:

$$
J_{g}=\frac{v}{v_{o}} \text {. }
$$

The other relationship that can be used is that for adiabatic gas compression, in which no heat transfer is allowed to occur. The pressure for this case is as follows:

$$
p_{a}=\frac{p_{o}}{J_{g}^{\gamma}}
$$

It can be shown that if a strain energy density per initial unit volume of gas is $W\left(J_{g}\right)$, then the relationship between $W$ and the current pressure $p$ is as follows:

$$
p=-\frac{\partial W}{\partial J_{g}} \text {. }
$$

Both the adiabatic and isothermal compression laws may be integrated to provide the following strain energy functions for pore gas:

$$
W_{i}=W_{0}-p_{o} \ln \left(J_{g}\right)
$$

and

$$
W_{a}=W_{0}+\frac{p_{o}}{\gamma-1}\left(J_{g}^{1-\gamma}-1\right) .
$$

We can use the incompressible matrix assumption to derive an expression for the relative volume of pore gas in a foam as follows:

$J_{g}=\frac{J-1}{\phi_{o}}+1$.

Once can substitute the expression in Equation 31 into the strain energy expressions of Equations 27 and 28 to calculate the strain energy per initial unit volume of gas in terms of the initial porosity and the macroscopic relative volume. To develop an expression for the pore gas strain energy per initial unit volume of the foam, one simply multiplies this expression by the initial porosity as follows:

$U_{g(a, i)}=\phi_{o} W_{(a, i)}\left(J_{g}\left(J, \phi_{o}\right)\right)$.

Similar but less rigorous developments have been made by others, but this model extension is rigorously tied to the initial foam porosity. This provides a stress contribution to the foam as follows:

$$
\boldsymbol{\sigma}_{g}=-p_{o} \mathbf{i} \begin{cases}\frac{1}{J-\left(1-\phi_{o}\right)} & \text { isothermal } \\ \frac{1}{\left[J-\left(1-\phi_{o}\right)\right]^{\gamma}} & \text { adiabatic }\end{cases}
$$

\section{CONCLUSIONS}

A strain energy density function for foamed rubber has been developed and demonstrated. It is a hybrid model, combining rigorously developed strain energy functions for isolated pores in an incompressible Mooney-Rivlin parent material with more phenomenological ad hoc strain energy function to capture initial stiffness, Young's modulus, and Poisson's ratio along with buckling phenomena.

The model has a feature that it becomes singular when the foam is compressed to the point where the relative volume is equal to the foam relative density. This is an approximation to observed physical response and ignores effects of matrix material compressibility which would serve to remove this singularity while preserving a very stiff behavior at large compressions.

Given that the developed constitutive model is only a strain energy density function, several extensions are possible. Two have been considered here.

The first model extension considered is associated with material aging and represents the effects of crosslink density change in a deformed storage state. This approach can produce observed permanent set and stiffness changes.

The second model extension considered here is the inclusion of pore gas compression effects. This concept has been considered by others, but is more rigorously developed here.

Several other model extensions are possible, including the use of developed equivalent isochoric invariants in more physically-based strain energy functions like the Arruda-Boyce model proposed in Arruda and Boyce (1993), viscoelasticity, and the Mullins effect.

\section{REFERENCES}

Braydon, A. D., Bardenhagen, S. G., Miller, E. A., and Seidler, G. T., December 2005, Simulation of the densification of real open-celled foam microstructures, JMPS, 53(12): p. 2638-2660.

Danielsson, M., Parks, D.M., and Boyce, M. C., 2004, Constitutive modeling of porous hyperelastic materials, Mech. of Mat. 36, 347-358.

Jemiolo, S. and Turteltaub, S., June 2000,Parametric model for a class of foam-like isotropic hyperelastic materials, $J$. Appl. Mech., v. 67, no. 2, p.248-254.

Kraynik, A.M., Nielsen, M.K., Reinelt, D.A., and Warren, W.E., 1998, "Foam Micromechanics," NATO Institute for 
Advanced Studies, Sandia Report SAND98-2454J, Albquerque, New Mexico.

Mooday, R., 2002, private communication, Los Alamos National Laboratory, Los Alamos, New Mexico.

Ogden, R.W., 1984, Non-linear elastic deformations, published by Ellis Harwood, Ltd., New York.

Tobolsky, A.V., 1960, Properties and Structure of Polymers. John Wiley and Sons, New York.

Arruda, E.M., and Boyce, M. C., 1993, A three-dimensional constitutive model for the large stretch behavior of rubber elastic materials, JMPS, 41(2):389-412. 